\title{
Downregulation of miR-21 inhibits EGFR pathway and suppresses the growth of human glioblastoma cells independent of PTEN status
}

\author{
Xuan Zhou ${ }^{1,2,7}$, Yu Ren ${ }^{3,7}$, Lynette Moore ${ }^{4}$, Mei Mei $^{3}$, Yongping You ${ }^{5}$, Peng Xu' ${ }^{1}$, Baoli Wang ${ }^{6}$, \\ Guangxiu Wang ${ }^{1}$, Zhifan Jia ${ }^{1}$, Peiyu Pu ${ }^{1}$, Wei Zhang ${ }^{4}$ and Chunsheng Kang ${ }^{1}$
}

MicroRNAs (miRNAs) are a class of endogenous small noncoding RNAs that regulate gene expression after transcription. Aberrant expression of miRNAs has been shown to be involved in tumorigenesis. We showed that miR-21 was one of the most frequently overexpressed miRNA in human glioblastoma (GBM) cell lines. To explore whether miR-21 can serve as a therapeutic target for glioblastoma, we downregulated miR-21 with a specific antisense oligonucleotide and found that apoptosis was induced and cell-cycle progression was inhibited in vitro in U251 (PTEN mutant) and LN229 (PTEN wildtype) GBM cells; xenograft tumors from antisense-treated U251 cells were suppressed in vivo. Antisense-miR-21-treated cells showed a decreased expression of EGFR, activated Akt, cyclin D, and Bcl-2. Although miR-21 is known to regulate PTEN and downregulation of miR-21 led to increased PTEN expression both endogenously and in a reporter gene assay, the GBM suppressor effect of antisense-miR-21 is most likely independent of PTEN regulation because U251 has mutant PTEN. Microarray analysis showed that the knockdown of miR-21 significantly altered expression of 169 genes involved in nine cell-cycle and signaling pathways. Taken together, our studies provide evidence that miR-21 may serve as a novel therapeutic target for malignant gliomas independent of PTEN status.

Laboratory Investigation (2010) 90, 144-155; doi:10.1038/labinvest.2009.126; published online 4 January 2010

KEYWORDS: miR-21; glioma; PTEN; Akt; EGFR

Malignant gliomas are the most common primary brain tumors with high mortality and morbidity. The prognosis for malignant gliomas has not significantly improved in the last four decades. A recent meta-analysis of 12 randomized clinical trials showed that the overall survival rate of highgrade gliomas was $40 \%$ at 1 year after surgical removal and only slightly higher, $46 \%$, after combined radiotherapy and chemotherapy. ${ }^{1}$ To develop more optimized and effective treatment strategies for malignant gliomas, it is critical to gain deeper understanding of the molecular mechanisms underlying gliomagenesis and to identify targets for therapeutic intervention.

The microRNAs (miRNAs) are a class of highly conserved small non-coding RNAs, approximately 22 nucleotides in length, that control gene expression through binding to the seed sequence at the $3^{\prime}$-UTR (untranslated region) of target mRNAs, resulting in translational repression or mRNA degradation. ${ }^{2}$ This regulatory mechanism was first shown in the developmental processes in worms, flies, and plants. ${ }^{3-5}$ Subsequently, miRNAs have been shown to have important roles in many physiological processes of mammalian systems by influencing cell apoptosis, proliferation, differentiation, development, and metabolism through regulation of critical signaling molecules including cytokines, growth factors, transcription factors, and pro-apoptotic and anti-apoptotic proteins. ${ }^{6-8}$ Increasing number of miRNAs have been identified in the human genome and they are collectively called the miRNome. ${ }^{9}$ Accumulating evidence shows the potential

\footnotetext{
${ }^{1}$ Department of Neurosurgery, Tianjin Medical University General Hospital, Laboratory of Neuro-oncology, Tianjin Neurological Institute, Tianjin, China; ${ }^{2}$ Department of Head and Neck Cancer, Tianjin Medical University Cancer Institute and Hospital, Tianjin, China; ${ }^{3}$ Tianjin Research Center of Basic Medical Science, Tianjin Medical University, Tianjin, China; ${ }^{4}$ Department of Pathology, University of Texas, MD Anderson Cancer Center, Houston, TX, USA; ${ }^{5}$ Department of Neurosurgery, Nanjing Medical University Affiliated Hospital, Nanjing, China and ${ }^{6}$ Key Lab of Hormone and Development, Institute of Endocrinology, Tianjin Medical University, Tianjin, China Correspondence: Dr C Kang, MD, Department of Neurosurgery, Tianjin Medical University General Hospital, Laboratory of Neuro-oncology, Tianjin Neurological Institute, Tianjin 300052, China. E-mail: kang97061@yahoo.com or Dr W Zhang, PhD, Department of Pathology, University of Texas, MD Anderson Cancer Center, Houston, TX 77030, USA. E-mail: wzhang@mdanderson.org
}

${ }^{7}$ These authors contributed equally to this work. 
involvement of altered regulation of miRNAs in initiation and progression in a wide range of human cancers. Altered expression profiles of miRNAs are associated with genetic and epigenetic alterations including deletion, amplification, point mutation, and aberrant DNA methylation. ${ }^{10}$ The miRNA cluster miR-15a-16, including $m i R-15 a$ and miR-16-1, is located near $13 \mathrm{q} 14$, a region that is characterized by a high frequency of deletion in chronic lymphocytic lymphoma. ${ }^{11}$ The expression of miR-15a and miR-16-1 were inversely correlated with the anti-apoptotic Bcl-2 gene expression in chronic lymphocytic leukemia and both miRNAs negatively regulated $\mathrm{Bcl}-2$ at the post-transcriptional level, resulting in the potentiation of the normal apoptotic response. ${ }^{12}$ In a mouse model of lymphoma, increased expression of the miR-17-92 cluster strongly accelerated lymphomagenesis and was the first functional evidence of an miRNA acting as a mammalian oncogene. ${ }^{13}$ Another example of oncogenic miRNAs is miR-29. Enforced miR-29 reduced the expression level of Mcl-1, an anti-apoptotic factor, to facilitate oncogenesis of cholangiocarcinoma. ${ }^{14}$ Some miRNAs function as tumor suppressor genes. Let-7 was reported to negatively regulate Ras and c-Myc expression after the let-7a-1 precursor construct was transfected into human colon tumor cell lines and expression of miR-34a induced apoptosis in neuroblastoma cells. ${ }^{15-17}$

Recent reports suggested that miR-21 functions as an oncogene in human cancers. Ciafrè et al ${ }^{18}$ profiled the expression of 245 miRNAs in 10 glioblastoma (GBM) cell lines and nine freshly resected GBM samples and observed that miR-21 was overexpressed in human brain tumors. ${ }^{18}$ It was shown that when miR-21 was suppressed, cell growth inhibition and caspase-dependent apoptosis were observed in A172, U87, LN229, and LN308 cells. ${ }^{19}$ Multiple target genes of miR-21 have been reported. It has been shown that miR-21 modulates breast cancer cell anchorage-independent growth through suppressing TMP1 expression. ${ }^{20}$ In human colorectal, breast cancer, and renal cell carcinoma, miR-21 contributes to invasion and metastasis cell by inhibiting Pdcd 4 mRNA at the post-transcription level. ${ }^{21-23} \mathrm{~A}$ recent study showed that miR-21 targets PTEN gene through a binding site on the $3^{\prime}$-UTR in hepatocellular carcinoma. ${ }^{24}$ PTEN has been shown to be a critical tumor suppressor gene that is commonly inactivated in GBM by deletion, mutation, or attenuated expression. ${ }^{25}$ Thus, increased expression of miR-21 may contribute to the attenuated expression of PTEN in GBM.

To further characterize the potential of miR-21 as a target for treating GBM and to clarify the role of PTEN in the response, we used both in vitro and in vivo systems to study the effect of miR-21 suppression in the PTEN wild-type (LN229) and PTEN mutant (U251) GBM cell lines. ${ }^{26}$ We found for the first time that downregulation of miR-21 inhibited the EGFR and Akt pathways and the anti-GBM effect was independent of PTEN status, suggesting that miR21 is a broader therapeutic target for GBM.

\section{MATERIALS AND METHODS Cell Culture Conditions}

The human U251 and LN229 glioblastoma cell lines were purchased from the Institute of Biochemistry and Cell Biology, Chinese Academy of Science. Human glioblastoma cell lines, TJ861, TJ905, and TJ899, were established and characterized in Laboratory of Neuro-oncology, Tianjin Neurological Institute. ${ }^{27}$ Human glioblastoma cell line, A172, and astrocytoma cell line, H4, were provided by Professor Jinhuan Wang (Tianjin First Central Hospital, China). All cell lines were maintained in Dulbecco's modified Eagle's medium (DMEM) (Invitrogen, Carlsbad, CA, USA) supplemented with $10 \%$ fetal bovine serum (Invitrogen), $2 \mathrm{mM}$ glutamine (Sigma, St Louis, MO, USA), 100 units of penicillin/ml (Sigma), and $100 \mu \mathrm{g}$ of streptomycin/ml (Sigma), at $37^{\circ} \mathrm{C}$ with $5 \% \mathrm{CO}_{2}$.

\section{Oligonucleotides and Cell Transfection}

The 2'-O-methyl (2'-OMe-) oligonucleotides were chemically synthesized by SBS Genetech (Beijing, China). The 2'-OMethyl oligos were composed entirely of $2^{\prime}-O$-methyl bases with the following sequences: scramble sequence $5^{\prime}$-AAGGC AAGCUGACCCUGAAGU- $3^{\prime}$ and $2^{\prime}-O-M e-m i R-215^{\prime}$-GUCA ACAUCAGUCUGAUAAGCUA- $3{ }^{\prime}{ }^{19}$ Oligonucleotides $(50 \mathrm{~nm} / \mathrm{l})$ were transfected into U251 cells at $70 \%$ confluence using Oligofectamine according to the manufacturer's instructions (Invitrogen).

\section{MicroRNA Array and Reverse Transcription (RT)-Real-Time PCR}

Total RNA was extracted from GBM cells (U251, TJ866, TJ905, TJ899, and A172) and human astrocytoma cell line H4 using Trizol reagent (Invitrogen) for miRNA profile examination. Two separate total RNA samples of antisense-miR-21-treated U251 cell and control U251 cell were prepared for mRNA expression profiling. The miRNA microarray was obtained from CapitalBio Corporation (Beijing, China).

The individual oligonucleotide probes were printed in triplicate on chemically modified glass slides in a $21 \times 21$ spot configuration of each subarray for miRNA microarray. All of the oligonucleotide probes were printed in triplicate on one microarray, and each of the four subarrays contained 16 controls (Zip5, Zip13, Zip15, Zip21, Zip23, Zip25, Y2, Y3, U6, New-U2-R, tRNA-R, hsa-let-7a, hsa-let-7b, hsa-let-7c, 50\% dimethyl sulphoxide (DMSO), and Hex).

Total RNA (FirstChoice Total RNA, Ambion, Austin, TX, USA) from normal brain was used as a control. The miRNAs were enriched from total RNA using a mirVana miRNA Isolation Kit (Ambion) and labeled with mirVana Array Labeling Kit (Ambion). Labeled miRNAs were used for hybridization on each miRNA microarray containing 509 probes in triplicate at $42^{\circ} \mathrm{C}$ overnight. A double-channel laser scanner (LuxScan 10K/A, CapitalBio) was used to scan the arrays and quantified using image analysis software 
(LuxScan3.0, CapitalBio). Raw data were normalized to the result of normal brain RNA and analyzed using the significance analysis of microarrays (SAM, version 2.1, http:// www-stat.stanford.edu/ tibs/SAM, Stanford University, CA, USA) software.

For transfected cells, reverse transcription (RT) reaction was conducted with the mirVana ${ }^{\mathrm{TM}}$ qRT-PCR miRNA detection kit (Ambion). The real-time PCR was carried out with the mirVana qRT-PCR miRNA detection kit (Ambion). Amplification reaction was performed using MJ-real-time PCR (Bio-Rad, Hercules, CA, USA) and the protocol was performed for 40 cycles at $95^{\circ} \mathrm{C}$ for $3 \mathrm{~min}, 95^{\circ} \mathrm{C}$ for $15 \mathrm{~s}$, and $60{ }^{\circ} \mathrm{C}$ for $30 \mathrm{~s}$. Both RT and PCR primer were purchased from Ambion. 5S RNA was used for normalization. Relative quantification was conducted using amplification efficiencies derived from cDNA standard curves and obtained relative gene expression. Data were shown as fold change $\left(2^{-\Delta \Delta \mathrm{Ct}}\right)$ and analyzed initially using Opticon Monitor Analysis Software V2.02 software (MJ Research, Waltham, MA, USA).

\section{Microarray Analysis}

Two separate total RNA samples were extracted from control and miR-21 knocked down U251 cells using Trizol reagent (Invitrogen). Samples were then sent to Beijing CapitalBio Corporation for processing. A total of $500 \mathrm{ng}$ of total RNA was processed for use on the microarray by using the Affymetrix GeneChip 3' IVT Express Kit (Affymetrix, Santa Clara, CA, USA) according to the manufacturer's recommended protocols. In brief, a first-stranded cDNA was synthesized from the total RNA sample using Superscript II Reverse Transcriptase (Invitrogen) and an oligo d (T) primer was linked with a T7 RNA polymerase binding site sequence. The amplified, labeled cRNA was produced using T7 RNA polymerase and biotinylated nucleotides. After removal of free nucleotides, cRNA yield was measured by UV260 absorbance with Nanodrop spectrophotometer (Gene, Carlsbad, CA, USA). The labeled cRNA was incubate with the $5 \times$ array fragmentation buffer at $94{ }^{\circ} \mathrm{C}$ for $35 \mathrm{~min}$ for fragmentation reaction and combined with hybridization internal positive control (ACTB, GAPD, LDHA, and RPS9) and positive control (5'-Hex-GTCAC ATGCGATGGATCGAGCTCCTTTATCATCGTTCCCACCTTA ATGCA- $3^{\prime}$ ) for DNA immobilization. After the signal amplified according to the standard protocols, arrays were scanned with the Affymetrix Model 3000 scanner and data were analyzed using GSEABase software (http://www. r-project.org/). To identify differentially expressed genes, signal values were transformed to logarithm base 2 and $z$-scores were calculated. A significant $P$-value was assigned to each $z$ score by $z$-test. Genes with $P$-value of $\leq 0.01$ were considered differentially expressed. Statistical analysis was conducted using MATLAB 7.0 (The MathWorks, Inc.).

\section{Gene Ontology and KEGG Pathway Analysis}

Genes with $P$-value of $<0.01$ were selected as differentially expressed genes and used for further analysis. Expression
Analysis Systematic Explorer (EASE) was used to analyze gene ontology and KEGG pathways. ${ }^{28}$ Overrepresentation of genes are present if a larger fraction of genes within that pathway is differentially expressed compared with all genes in the genome. An EASE score of $\leq 0.05$ was used as a cutoff.

\section{MiR-21 Detection by In Situ Hybridization}

Using antisense locked nucleic acid (LNA)-modified oligonucleotides probe, in situ hybridization was performed with In situ hybridization kit (Boster, Wuhan, China). LNA/DNA oligos contained locked nucleic acids at eight consecutive centrally located bases (indicated by the underline) and had the following sequences: LNA-miR-21 5'-TCAACATCAGT CTGATAAGCTA- $3^{\prime}$. At $72 \mathrm{~h}$ after transfection, U251 cells were fixed with freshly prepared $4 \%$ paraformaldehyde (containing $0.1 \%$ DEPC). The in situ hybridization detection of miR-21 in U251 GBM cells were conducted according to the protocol of the manufactures. The fixed U251 cells were incubated with $20 \mu \mathrm{l}$ LNA-miR-21 hybridization solution at $42{ }^{\circ} \mathrm{C}$ for $16 \mathrm{~h}$, and $\mathrm{Cy} 3$-avidin was used to label miR-21 at a concentration of $0.5 \mathrm{mg} / \mathrm{ml}$. Nuclei were counterstained with DAPI karyotyping kit (Genmed, Boston, MA, USA) and visualized using FluoView Confocal Laser Scanning Microscopes-FV1000 (Olympus, Tokyo, Japan) and analyzed using IPP5.1 (Olympus).

\section{Cell Proliferation Assay}

U251 and LN229 cells were seeded into 96-well plates at 4000 cells per well. After transfection as described previously, $20 \mu \mathrm{l}$ of MTT $(5 \mathrm{~g} / \mathrm{l})$ was added into each well at each day of consecutive 6 days after treatment and the cells were incubated for additional $4 \mathrm{~h}$, and the supernatant was then discarded. Finally, $200 \mu \mathrm{l}$ of DMSO was added to each well to dissolve the precipitate. Optical density (OD) was measured at the wavelength of $570 \mathrm{~nm}$. The data are presented as the mean \pm s.d., which are derived from triplicate samples of at least three independent experiments.

\section{Cell-Cycle Analysis}

For cell-cycle analysis using FCM (flow cytometry), transfected and control cells in the log phase of growth were harvested, washed with PBS, fixed with $90 \%$ ethanol overnight at $4{ }^{\circ} \mathrm{C}$, and then incubated with RNase at $37^{\circ} \mathrm{C}$ for $30 \mathrm{~min}$. Nuclei of cells were stained with propidium iodide for additional $30 \mathrm{~min}$. A total of $10^{4}$ nuclei were examined in a FACS Calibur flow-cytometer (Becton Dickinson, Franklin Lakes, NJ, USA). Samples were analyzed using flow cytometry for FL-2 area and DNA histograms were analyzed using Modifit software. Experiments were performed in triplicate. Results are presented as percentage of cell in phase.

\section{Apoptosis Assays Using Annexin Staining and TUNEL Method}

Parental and transfected cells in the log phase of growth were harvested and collected by centrifugation for $5 \mathrm{~min}$ at $500 \mathrm{~g}$ 
and cells were then resuspended at a density of $1 \times 10^{6}$ cells $/ \mathrm{ml}$. For the Annexin V assay, the annexin V-Cy3-labeled Apoptosis Detection Kit (Abcam, Cambridge, MA, USA) was used. The apoptotic cells were detected and quantified using FACSCalibur (Becton Dickinson, San Jose, CA, USA). The data obtained were analyzed using CellQuest software.

The apoptotic cell death in the tumor specimens of mouse models from the in vivo study was examined by TUNEL method using an in situ cell death kit (Roche, Indianapolis, IN, USA). For detecting miRNA-21 expression and apoptotic cells simultaneously, in situ hybridization was performed as described above, and then apoptosis was detected using TUNEL method. Positive cells were visualized using fluorescence microscopy. The reaction mixture was incubated without enzyme in a control coverslip to detect nonspecific staining. Nuclei were counterstained with DAPI karyotyping kit (Genmed) and visualized using FV-1000 laser scanning confocal biological microscopes and analyzed using IPP5.1 (Olympus).

\section{Western Blot Analysis}

Parental and transfected cells were thrice washed with prechilled phosphate-buffered saline (PBS). The cells were then solubilized in $1 \%$ Nonidet P-40 lysis buffer $(20 \mathrm{mM}$ Tris, $\mathrm{pH}$ $8.0,137 \mathrm{mM} \mathrm{NaCl}, 1 \%$ Nonidet P-40, $10 \%$ glycerol, $1 \mathrm{mM}$ $\mathrm{CaCl}_{2}, 1 \mathrm{mM} \mathrm{MgCl}_{2}, 1 \mathrm{mM}$ phenylmethylsulfonyl fluoride, $1 \mathrm{mM}$ sodium fluoride, $1 \mathrm{mM}$ sodium orthovanadate, and a protease inhibitor mixture). A total of $40 \mu \mathrm{g}$ lysates were subjected to SDS-PAGE on 8\% SDS-acrylamide gel. Separate proteins were transferred to PVDF membranes (Millipore, Bedford, MA, USA) and incubated with primary antibodies against Akt-2 (Santa Cruz, Santa Cruz, CA, USA), ${ }^{29}$ pAKT (for Ser473, Santa Cruz), PTEN, EGFR, STAT3, and Bcl-2 (Zhongshan Bio Corp., Beijing, China) followed by incubation with an HRP-conjugated secondary antibody (Zymed, San Francisco, CA, USA). The specific protein was detected using a SuperSignal protein detection kit (Pierce, Rockford, IL, USA). The membrane was stripped and re-probed with a primary antibody against $\beta$-actin (Santa Cruz).

\section{DNA Constructs and Luciferase Assay}

Luciferase activity assay-related constructs were made by ligating PTEN 3'-UTR fragments (approximately $500 \mathrm{bp}$ ) containing the predicted binding sites into luciferase reporter vector (pGL3).

PCR was performed to amplify a fragment containing the miR-21 target sequence using human DNA as the template. The primers used for PTEN $3^{\prime}$-UTR were $5^{\prime}$-ACTCTAGA GTCGACACCACTGACTCTGATC- $3^{\prime}$ and $5^{\prime}$-ACTCTAGACA TGACACAGCTACACAACC- $3^{\prime}$. The product was digested using $X b a \mathrm{I}$ enzyme and afterward ligated with $X b a I$-treated pGL3-control vector containing the SV40 promoter. The ligated product was transformed into E. coli JM109 and colony PCRs were used to screen for the clones harboring the forwardly oriented insert using the antisense primer of PTEN and a sense primer of the vector (5'-AGGAGTTGTGTT
TGTGGACG-3'). The desired construct was subsequently sequenced by Invitrogen.

For luciferase reporter experiments, the pGL3-PTEN-3UTR construct, which contains the putative binding site for miR-21 of the 3-UTR of the PTEN, was amplified by PCR from human genomic DNA and inserted into the pGL3 control vector (Promega, Madison, WI, USA), using the XbaI site immediately downstream from the stop codon of luciferase in the reporter gene vector. U251, A172, and H4 cells were plated $\left(2 \times 10^{6}\right.$ cells per well $)$ in six-well plates. After transfection, the cells were split into 96 -well plates in duplicate and harvested for luciferase assays $24 \mathrm{~h}$ later using a luciferase assay kit (Promega).

\section{U251 Xenograft Tumor Assay}

Female immune-deficient nude mice (BALB/C-nu), 5 weeks old, were purchased from the animal center of the Cancer Institute of Chinese Academy of Medical Science, and were bred at Compare Medicine Center, Tianjin Medical University. All experimental procedures were performed according to Tianjin Medical University policies.

In all, four mice were injected subcutaneously with $1 \times 10^{6}$ of U251 GBM cells, in a volume of $50 \mu \mathrm{l}$ of PBS pre-mixed with equal volume of matrigel matrix (Becton Dickinson). Mice were monitored daily and three out of four mice formed tumors subcutaneously. When the tumor size reached approximately $5 \mathrm{~mm}$ in length, the tumors were surgically removed, cut into pieces of $1-2 \mathrm{~mm}^{3}$ and re-seeded into the left inguinal region of 30 mice. When the diameter of the subcutaneous tumor reached $7 \mathrm{~mm}$ the mice were divided into three groups (10 mice per group) randomly: U251 control group, U251 scramble oligonucleotide (ODN)-treated group, and U251 As-miR-21-treated group. A mixture of $20 \mu \mathrm{l}$ oligofectamine and ODN (50 nmol/l) mixture was injected into the xenograft tumor model in a multi-site injection manner. Mice in the U251 control group received $10 \mu \mathrm{l}$ of PBS only. A second administration was conducted on day 3 . The tumor volume was measured with a caliper every 3 days, using the formula volume $=$ length $\times$ width $^{2} / 2$.

At the end of the 22-day observation period, the mice bearing xenograft tumors were killed and the tumor tissues were removed for formalin fixation and preparation of paraffin-embedded sections.

\section{Immunofluorescence and Immunohistochemistry Staining}

The paraffin-embedded tissue sections were used for examination of PTEN, EGFR, p-AKT, AKT-2, Ki67, cyclin D1, Bcl-2 expression, and HE staining. For immunofluorescence staining, transfected cells were seeded on coverslips and fixed with $4 \%$ paraformaldehyde (PFA, Sigma), treated with $3 \% \mathrm{H}_{2} \mathrm{O}_{2}$ for $10 \mathrm{~min}$ and incubated with the antibodies described above overnight at $4{ }^{\circ} \mathrm{C}$. FITC- or TRITC-labeled secondary antibody (1:200 dilutions) was added for $2 \mathrm{~h}$ at $37^{\circ} \mathrm{C}$. DAPI reagent was used to stain the 
U251 cell nuclei and the cells was visualized using FV-1000 laser scanning confocal microscopes and analyzed using IPP5.1 (Olympus). For immunohistochemistry study, sections were incubated with primary antibodies (1:200 dilutions) overnight at $4{ }^{\circ} \mathrm{C}$, followed by biotin-labeled secondary antibody (1:100 dilutions) for $1 \mathrm{~h}$ at room temperature. Sections were then incubated with $\mathrm{ABC}$-peroxidase and $\mathrm{DAB}$ (diaminobenzedine), counterstained with hematoxylin, and visualized using light microscope.

\section{Statistical Analysis}

Data were expressed as means \pm s.e. Statistics was determined using ANOVA, $\chi^{2}$ test, or Student's $t$-test using SPSS11.0 (Windows). Statistical significance was determined as $P<0.05\left(^{*}\right)$ or $\left.P<0.01{ }^{* *}\right)$.

\section{RESULTS}

\section{MiR-21 was Overexpressed in Human Glioblastoma Cell Lines}

We first profiled miRNA expression in five glioblastoma cell lines, one astrocytoma cell line, and one normal brain tissue. Our analysis showed that 8 of the 435 human miRNAs $(1.84 \%)$ were overexpressed with a greater than twofold increase and 18 of $453(3.68 \%)$ showed a greater than twofold reduction in all glioma cell lines (Figure 1a and b). Among the increased miRNAs, miR-21 showed the most significant increase relative to normal brain tissue (7.0-fold). Other increased miRNAs include miR-221, miR-222, and miR-23a. The miR-21 expression results from microarrays were confirmed with quantitative RT-PCR assays (Figure 1c). MiR-21 was chosen for further study because it was the most prominently overexpressed miRNA in glioma.

\section{Antisense-miR-21Suppressed U251 Glioma Cell Proliferation and Induced Apoptosis}

To evaluate the significance of miR-21 overexpression in glioma cells, we used a loss-of-function antisense approach. An As-miR-21 oligonucleotide (ODN) was used to knock down miR-21 expression in U251 and LN229 cells. RT-realtime PCR results determined that the relative expression level of miR-21 in As-miR-21 ODN-treated U251 cell was 6.25\% $(P<0.01)$ and $12.5 \%$ for LN229 cells $(P<0.01)$ compared with their control cells, respectively (Figure 2a). In addition, LNA-based in situ hybridization showed that transfection of a scrambled ODN had no effect on miR-21 expression. In contrast, the cy3 red fluorescence signal in As-miR-21transfected U251 cells was lower (Figure 2b). These data

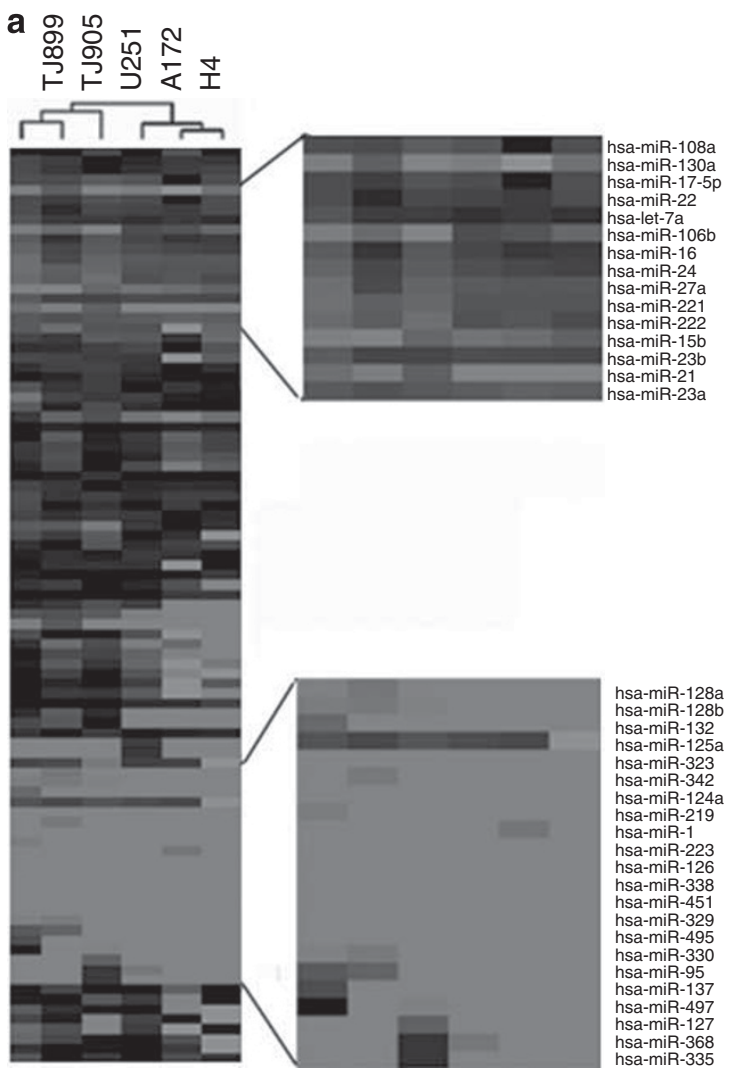

b

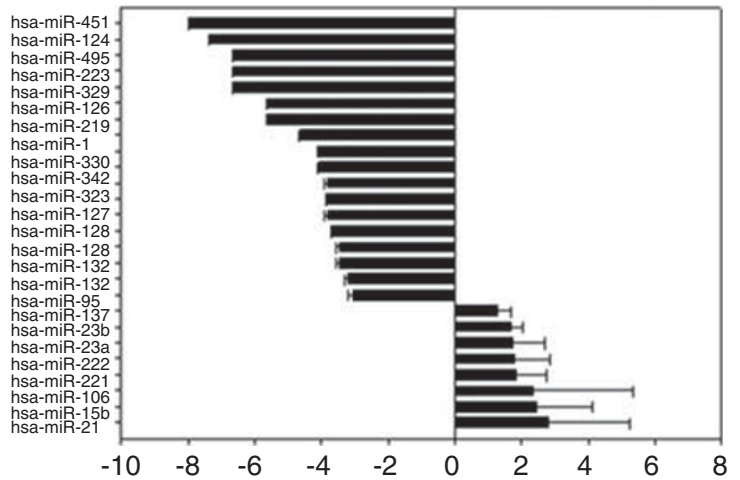

Log2 (Expression in Glioma and Glioblastoma cell line/Ambion normal brain)

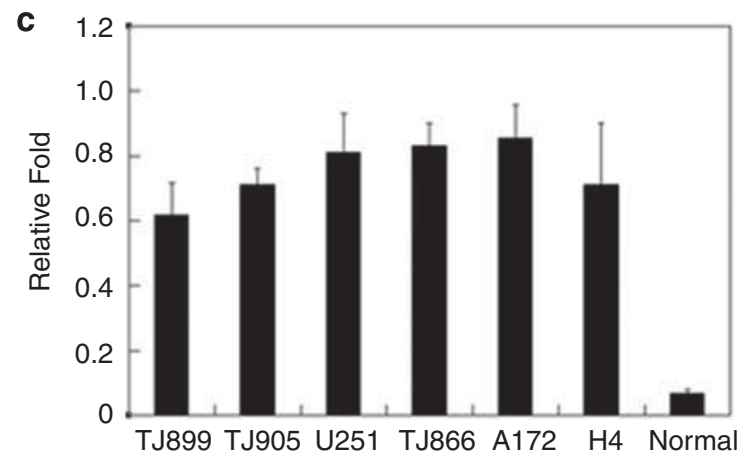

Figure 1 miR-21 is overexpressed in glioma cells. (a) Cluster analysis of miRNA expression profile across human glioblastoma cell lines. Glioma cell lines were presented in columns, miRNAs in rows. (b) The 26 most upregulated (right) and downregulated (left) miRNAs in six human glioma and globlastoma cell lines with respect to Ambion normal brain total RNA. The relative expression of miRNAs level is present in log2 transformed for each glioblastoma cell line. (c) RT-real-time PCR shows miR21 overexpression in glioma cell lines. U6 snRNA was used as a loading control. 
a

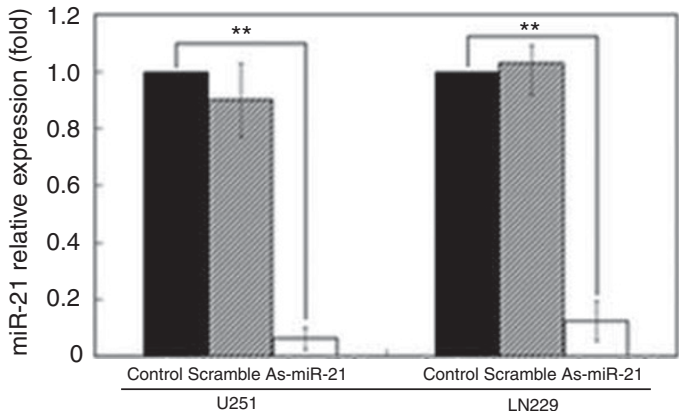

b
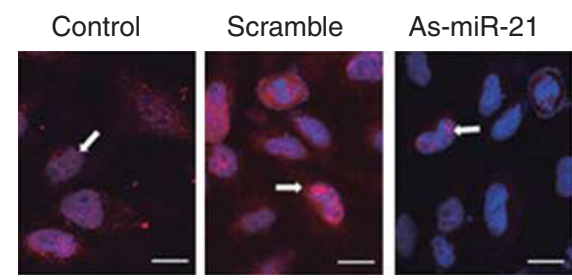

d
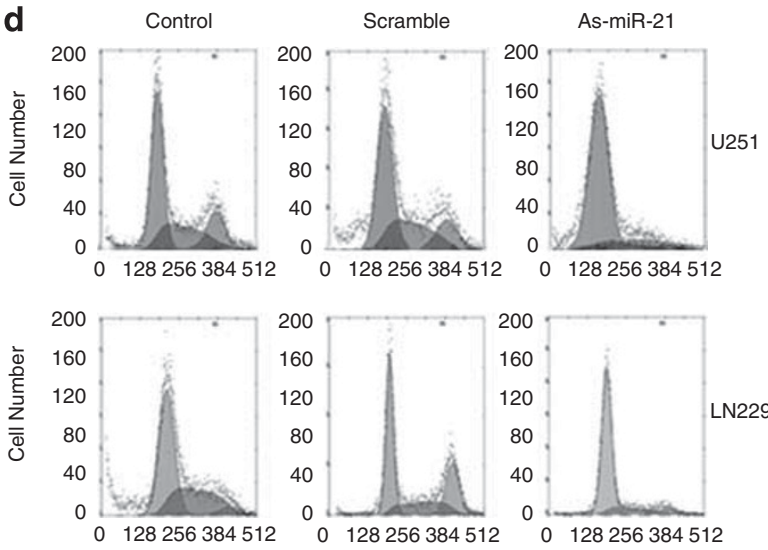

DNA content

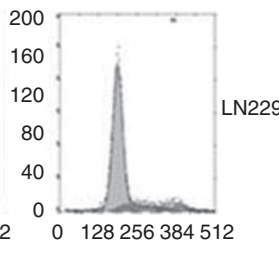

e
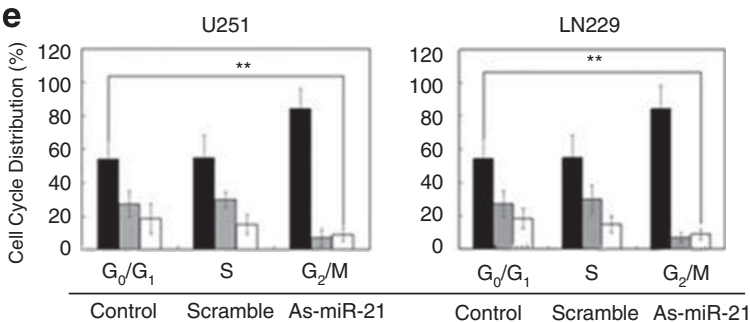
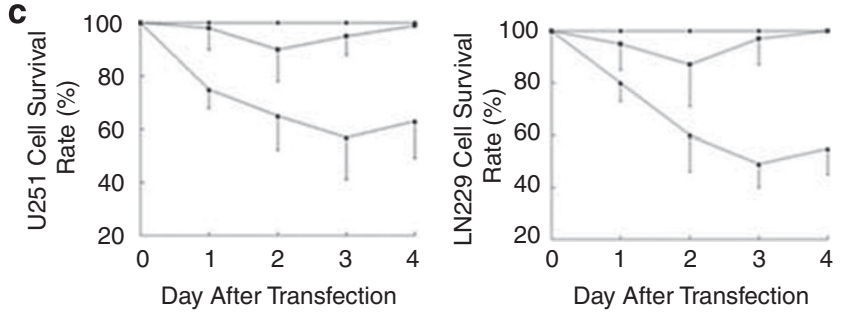

f
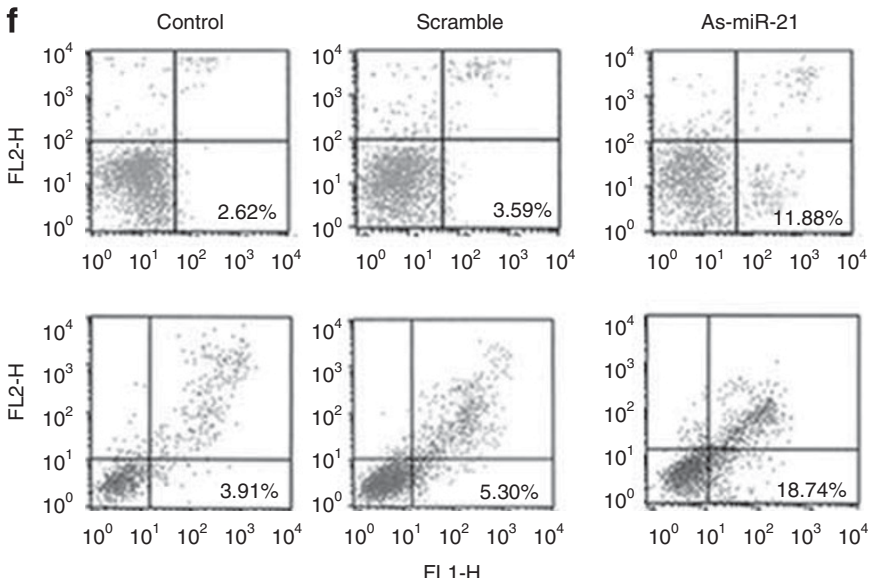

g
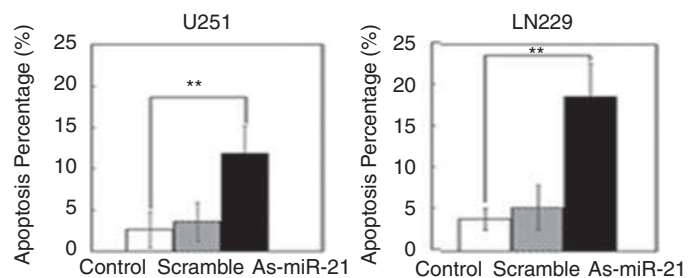

Figure 2 The effect of miR-21 knockdown on U251 and LN229 GBM cell proliferation. (a) miR-21 expression was suppressed in U251 and LN229 cells using RT-real-time PCR. (b) In situ examination of miR-21 expression in U251 cells. Arrows highlight miR-21 in situ expression in U251 cells. Bar $=20 \mu \mathrm{m}$. (c) MTT cell proliferation assay. miR-21 knockdown in U251 and LN229 GBM inhibits cell proliferation. (d) Cell-cycle profiles after PI staining. miR-21 knockdown induced $\mathrm{G}_{1}$ arrest in both U251 and LN229 GBM cells. (e) Graphical representation of the cell-cycle profiles in (d). As-miR-21 and scramble ODN-transfected U251 and LN229 GBM cells were analyzed using FCM to determine cell-cycle status. $\chi^{2}$ Test was performed. ${ }^{* *} P<0.01$. (f) Analysis of apoptosis using Annexin V staining. MiR-21 knockdown led to U251 and LN229 GBM cell apoptosis. (g) Graphical representation of (f). As-miR-21 and scramble ODN-transfected U251 and LN229 GBM cells were analyzed using FCM to determine cell apoptosis. One-way ANOVA was performed. ${ }^{\star \star P}<0.01$.

suggested that As-miR-21 can specifically inhibit the endogenous miR-21 expression in U251 and LN229 cells.

Cell viability was measured in ODN-transfected cells in up to 4 days after treatment. As-miR-21 ODN-treated cells showed a significant decrease in viability compared with control ODN-treated cells or untreated cells (Figure 2c). We found that the growth-inihibitory effect of decreased miR-21 reached maximum at 3 days after transfection. The lowest survival rate was $57 \pm 16 \%$ for U251 cell and $49 \pm 9 \%$ for LN229 cell. 
To further analyze whether decreased viability was a result of cell-cycle arrest, the cell-cycle distribution was analyzed using FCM. At $72 \mathrm{~h}$ after transfection, FCM analysis showed a $20 \%$ increase in $\mathrm{G}_{1}$-phase cells and a $25 \%$ decrease in S-phase cells in As-miR-21-treated U251 cells $\left(\chi^{2}=14.160, P<0.01\right)$. In miR-21 knocked down LN229 cells, the population of $\mathrm{G}_{1}$ phase increased 30\% compared with control and scramble ODN-treated cells $\left(\chi^{2}=35.677, P<0.01\right)$. There was no statistical difference in $\mathrm{G}_{2} / \mathrm{M}$-phase population among the three groups for both cell lines (Figure $2 \mathrm{~d}$ and e). These findings suggest that overexpressed miR-21 is required to regulate the $\mathrm{G}_{1}$-to-S phase transition in different $\mathrm{GBM}$ cell lines. Our studies are consistent with a previous observation that showed a $21-25 \%$ increase in G1 phase in the U251 cell line by miR-21 knockdown. ${ }^{30}$

We also analyzed the effect of decreased miR-21 on apoptosis by conducting Annexin V and PI double staining. The Annexin V-positive early-phase apoptotic cells were significantly increased in cells transfected with AS-miR-21 (11.88\% for U251 cells and $18.74 \%$ for LN229 cells) when compared with parental cells and cells transfected with scrambled ODN $(2.62 \%$ for U251 control and 3.91\% for LN229 cell; $P<0.01$; Figure $4 \mathrm{f}$ and g). Apoptotic nuclei were almost 7\% higher in the LN229 cell line compared with U251 cells. However, there was no significant difference in PI-positive cells, which is consistent with the lack of change in sub$\mathrm{G}_{1}$-phase cells in our FCM analysis.

\section{As-miR-21 Blocked EGFR and Akt Activation and Suppressed STAT3 Expression}

Computational analysis predicts that PTEN is a target gene for miR-2 $1^{24,31}$ and the Akt pathway is regulated by PTEN. ${ }^{29}$ PTEN inactivation and EGFR and Akt activation frequently occur in glioblastomas. ${ }^{32}$ Thus, one potential function of elevated miR-21 may be to downregulate the PTEN tumor suppressor gene. However, our analysis above showed that blocking miR-21 inhibited both U251 cells that have mutant PTEN and LN229 cells that have wild-type PTEN. A previous study showed that decrease of miR-21 also suppressed U87 cells that have no PTEN expression. ${ }^{19}$ Thus, we first examined whether As-miR-21 had any effect on the expression of a set of proteins that are important for glioma proliferation and survival. Both Western blotting and immunofluorescence assays were used. The level of endogenous PTEN protein increased and phosphorylated Akt decreased in both U251 and in LN229 GBM cells upon inhibition of miR-21. In addition, EGFR, cyclin D1, and Bcl-2 were also decreased, consistent with blocked $G_{1} / S$ transition and early apoptosis (Figure $3 a$ and b). Furthermore, we also observed a decrease in STAT3 protein in both LN229 and U251 GBM cells.

The observation that endogenous mutant PTEN was increased in the As-miR-21-treated U251 cells and wild-type PTEN was increased in the As-miR-21-treated LN229 cells provided evidence that miR-21 indeed regulates its target
PTEN mRNA in glioma cells. To further test this hypothesis, we generated a reporter gene construct pGL3-PTEN-3-UTR that contains the putative miR-21 binding site. ${ }^{24,31,33}$ The pGL3-PTEN-3'-UTR construct and pGL-3 control plasmid were separately co-transfected with AS-miR-21 or control ODN into U251, A172 (PTEN deleted), and H4 (PTEN status was not clear) glioma cells followed by measurement of luciferase activity in the transfected cells at 3 days after transfection. The results showed that the AS-miR-21 caused a four- to sixfold decrease in luciferase activity in pGL3-PTEN$3^{\prime}$-UTR-transfected cells (Figure $4 \mathrm{a}$ and b). Thus, the miR-21 binding site in $3^{\prime}$-UTR of PTEN gene is functional.

\section{Decrease of miR-21 by AS-miR-21 Altered Cellular Pathway Genes in U251 Cells}

Whereas the inhibition of gene expression by microRNAs is well understood, the mechanism of increased gene expression by microRNAs is less known, although some microRNAs have been shown to function as enhancers. To understand the effect of miR-21 on EGFR, cyclin D, and Bcl-2 expression, we performed a microarray analysis and KEGG pathway analysis to gain insight into the differences in gene expression before and after miR-21 knockdown in U251 cells. KEGG analysis comparing differentially expressed genes in miR-21 knockdown U251 cells showed that downregulation of miR-21 affected a number of pathways including 'cell cycle $(P=0.000)$ ', 'Ubiquitin-mediated proteolysis $(P=0.000)$ ', 'AminoacyltRNA biosynthesis $(P=0.000)$ ', 'Small cell lung cancer $(P=0.010)$ ', 'Insulin signaling pathway $(P=0.024)$ ', 'Adherens junction $(P=0.037)$ ', 'Renal cell carcinoma $(P=0.045)$ ', 'p53 signaling pathway $(P=0.049)$ ', and 'MAPK signaling pathway $(P=0.049)$ '. Among these, BID, FAS, PRS6, PTEN, and SOCS4 were upregulated genes with miR21 inhibition. Alteration of ubiquitin-mediated proteolysis may explain increased expression of some oncogenic proteins, such as EGFR, by miR-21.

\section{As-miR-21 Treatment Suppresses Glioblastoma Growth in Xenograft Model}

The in vitro experiments suggest that miR-21 is a potential target for therapy in GBM. To further confirm this, we performed a proof-of-principle experiment using a U251 glioma xenograft model and a lipofectamine-mediated gene therapy approach. Subcutaneous tumors, approximately $5-6 \mathrm{~mm}$ in length, were established in the right groin of 30 mice. In all, 10 mice were challenged by in situ injection of PBS as control group, 10 mice were treated with lipofectamine-mediated scrambled oligonucleotide as the second control group, and 10 mice were treated with lipofectamine-mediated AS-miR21 as the treatment group. At the beginning of treatment, the mean tumor volume of the mice in control, scramble, and treated groups was 82,90 , and $85 \mathrm{~mm}^{3}$, respectively, with no statistically significant difference among these three groups. The mice were monitored every 3 days for 3 weeks, and the tumor volume of mice in each group was measured and 
a

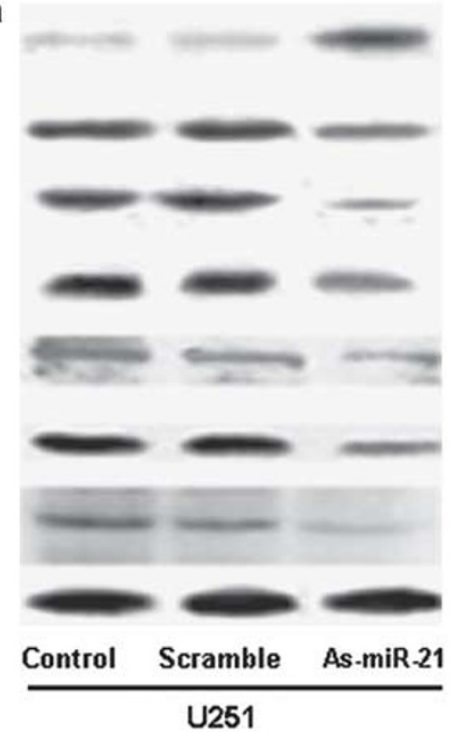

b
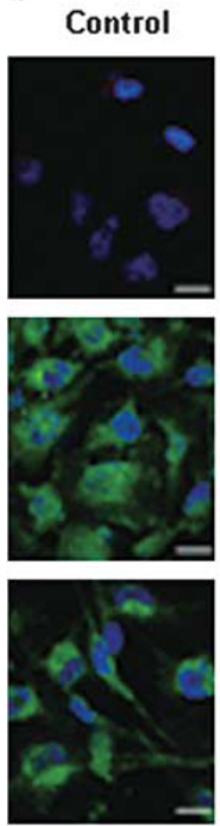
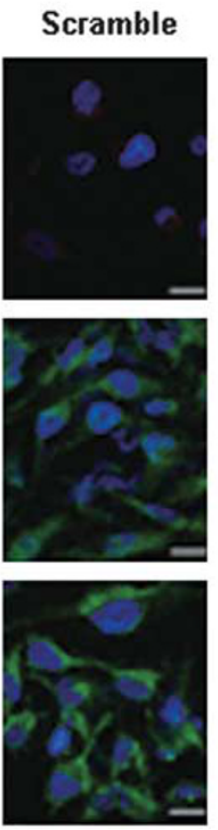

As-miR-21
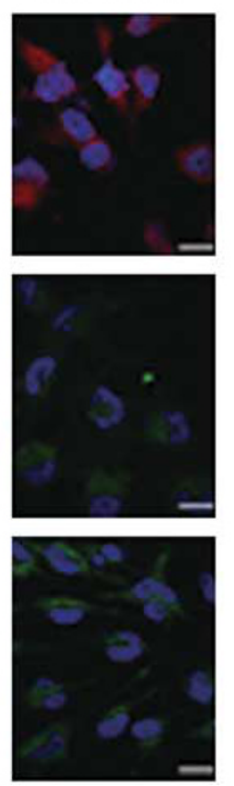

PTEN

P-AKT

AKT-2

EGFR

Cyclin D1

BcL-2

STAT-3

$\beta$-Actin

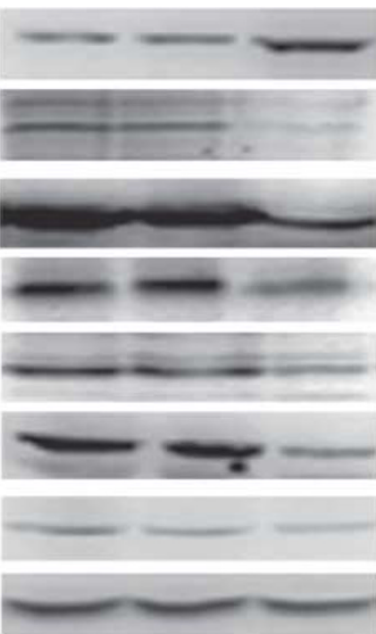

Control Scramble As-miR-21

LN229

PTEN
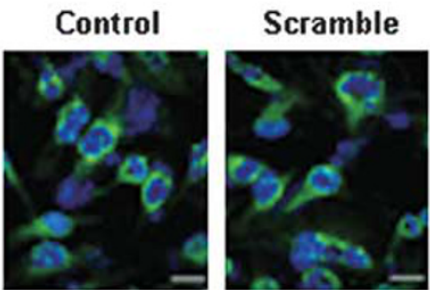

As-miR-21

EGFR
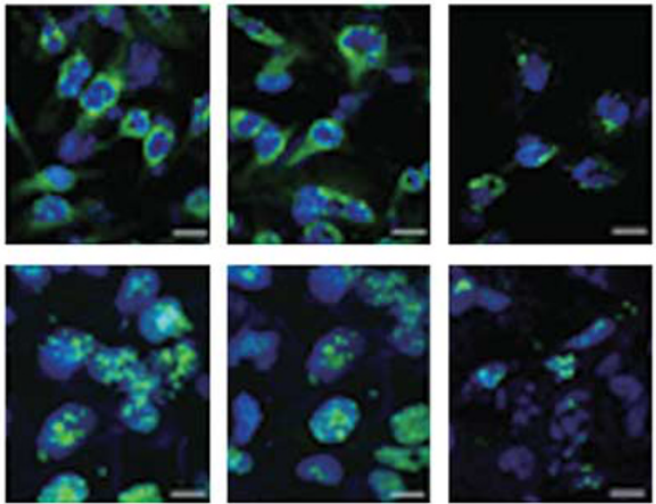

AKT-2

P-AKT
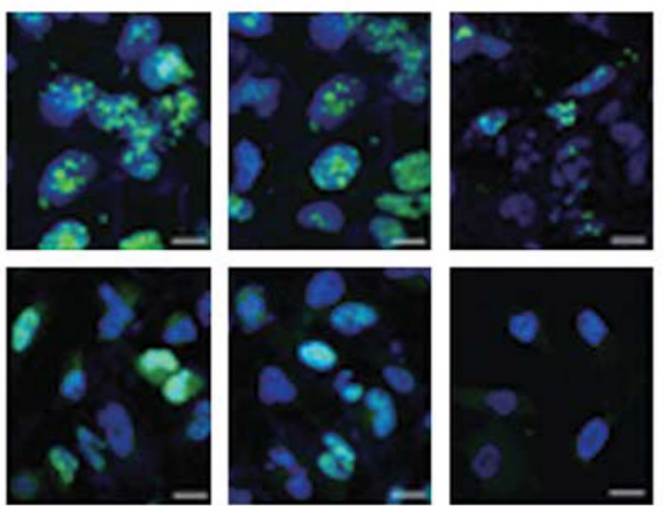

Ki67
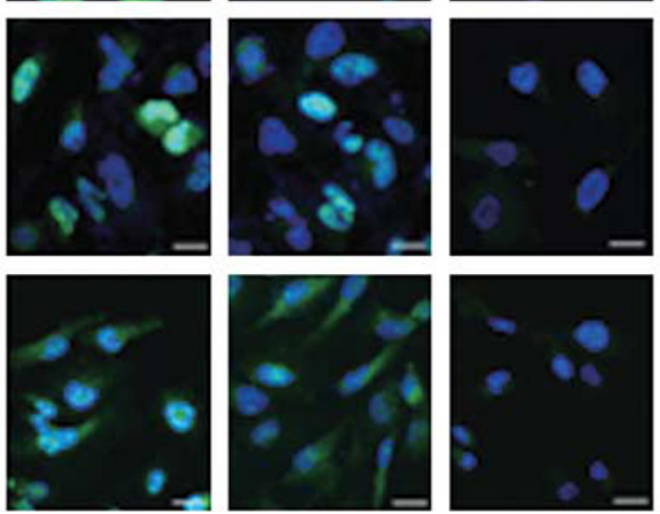

BcL-2

CyclinD1

Figure 3 As-miR-21 blocked EGFR and Akt activation and suppressed STAT3 expression. (a) Western blot showing that PTEN expression was increased in U251 and LN229 GBM cells. pAkt, AKT-2, EGFR, cyclin D1, Bcl-2, and STAT3 expression were suppressed simultaneously. (b) Immunofluorescence staining assay: PTEN expression was increased in U251 GBM cells. pAkt, AKT-2, EGFR, cyclin D1, and Bcl-2 expression were suppressed simultaneously. Bar $=30 \mu \mathrm{m}$.

compared. Significant decrease in tumor volume was only observed in the As-miR-21 treatment group (Figure 5a).

We collected the tumors and evaluated miR-21 expression by in situ hybridization and cellular apoptosis using TUNEL assay. Whereas miR-21 was highly expressed in control and scramble-treated U251 xenograft tumors, no apoptotic nuclei were found. In contrast, in the As-miR-21-treated xenograft tumors, miR-21 expression was markedly decreased and abundant apoptotic nuclei were observed (Figure 5b). In addition, tumors derived from control and scramble-treated 

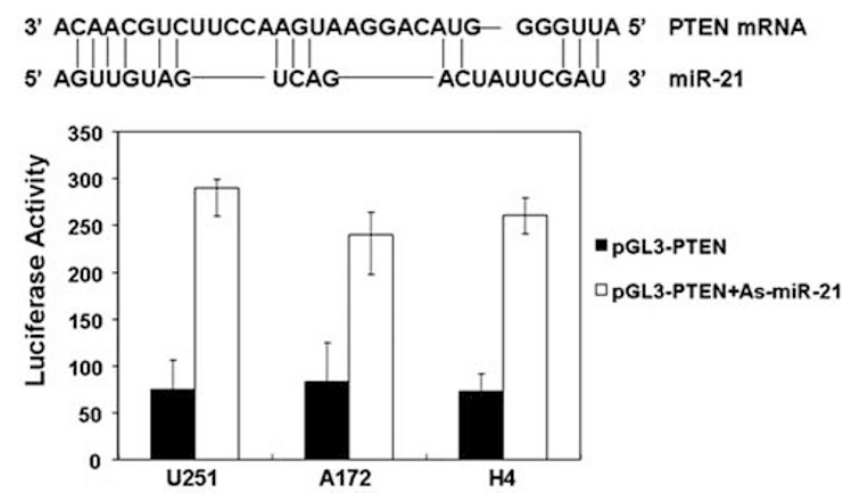

Figure 4 mIR-21 inhibits the PTEN $3^{\prime}$-UTR. (a) Schematic representation of the miR-21 target site beginning at nucleotide 23 of the PTEN-3'-UTR. (b) Luciferase activity was significantly increased by miR-21 knockdown in U251, LN229, and A172 GBM cells. The $t$-test was performed. ${ }^{*} P<0.01$.

cells revealed increased micro-vessel numbers and deeper chromatin staining compared with the As-miR-21-treated cells, as determined using HE stain (Figure $5 \mathrm{c}$ ). Consistent with the in vitro results, in the As-miR-21-treated tumors, we observed decreased expression of phosphorylated Akt as well as EGFR, AKT-2, Ki67, Bcl-2, and cyclin D1 (Figure 5c).

\section{DISCUSSION}

\section{MiR-21 is Overexpressed in Human GBM Cell Lines}

MiR-21 has been identified as one of the most overexpressed microRNAs in a number of medium-scale and large-scale profiling experiments designed for the detection of miRNAs dysregulated in human cancers. ${ }^{22-25}$ The first indication of the aberrant expression of miR-21 came from the miRNA profiling of human glioblastoma. ${ }^{18}$ Compared with normal brain tissue, miR-21 relative expression was 7 - to 11 -folds in low-grade astrocytomas, anaplastic astrocytomas, and GBMs. ${ }^{34}$ The present profile data further confirmed increased expression of miR-21 across GBM and astrocytoma cell lines. MiR-21 expression status was further validated using RT-real-time PCR in all six cell lines. Although GBM cell lines have extremely diverse genetic background, universal overexpression of miR-21 in Ciafre's study and the GBM cell lines (TJ899, TJ866, and TJ905) established in our lab strongly indicates that miR-21 has a robust role in glial cell cancerous transformation., ${ }^{2,18}$

\section{MiR-21 Suppresses GBM Cell Growth Independent of PTEN Status}

There is just partial complementarity between miRNAs and their targets in animal cells, and several computational algorithms predict hundreds of mRNAs as possible targets for miR- $21 ;{ }^{35}$ however, relatively few have been experimentally validated in GBM. Post-transcriptional downregulation of tumor suppressor PDCD4 by miR-21 stimulated T98G GBM cell growth and anti-apoptotic effect. ${ }^{36} \mathrm{MiR}-21$ also regulated
RECK and TIMP-3 gene to promote human cancer cell (A172, U87, LN229 GBM cells, Hela human cervix carcinoma cell, and MCF-7 breast cancer cell) anti-apoptosis, migration, and invasiveness effect by activating matrix metalloproteinases (MMPs). ${ }^{37}$ Furthermore, miR-21 post-transcriptional inhibiting leucine rich repeat interacting protein 1 (LRRFIP1) contributes to VM-26 resistance in U373 MG cell. ${ }^{2,38}$

PTEN tumor suppressor gene has been validated as an miR-21 target in human cholangiocarcinoma and hepatocellular carcinoma cells. ${ }^{24,33}$ This is particularly interesting as up to $80 \%$ of GBMs have a PTEN deficiency, and the prognosis of GBM can be predicted based on the levels of expression of PTEN. ${ }^{39,40}$ Loss of functional PTEN leads to increased activity of the Akt and the mammalian target of rapamycin (mTOR) kinase pathways, which can promote both tumor cell survival and proliferation through phosphorylation and activation of several downstream mediators. ${ }^{41}$ Thus, we initially hypothesized that GBM cells with wild-type PTEN should have a better response to As-miR-21 treatment compared with PTEN mutant or deleted GBM cells. Surprisingly, we observed similar growth-inhibitory effects in both U251 and LN229 cells using MTT assay, suggesting that some of the effects of miR-21 inhibition may be independent of PTEN.

To correlate the growth-inhibitory effects with anti-tumor effects of As-miR-21, we used a U251 GBM xenograft model. Successful inhibition of miR-21 inhibited U251 xenograft tumor proliferation and induced apoptosis in the tumor. These in vivo findings could indicate that miR-21 targeting therapy has great clinical usage potential independent of PTEN status. It was also reported that combined miR-21 inhibition with S-TRAIL led to synergetic cytotoxicity to suppress A172 and U87 GBM cell growth in vitro and U87 intracranial tumors in vivo. ${ }^{42}$

Consistent with its effects in xenograft tumors, As-miR-21 treatment promoted apoptosis in both U251 and LN229 cell lines. Decreased levels of the anti-apoptotic Bcl-2 were observed, suggesting that targeting miR-21 could suppress cell growth through the mitochondrial apoptosis pathway. ${ }^{43}$ MRNA expression profile scanning data from As-miR-21treated U251 cells strongly indicated that BID, FAS, PRS6, and SOCS4 tumor suppressor genes were upregulated. FAS gene has a central role in programmed cell death by sequential activation of caspase- 8 and caspase- 3 in both the normal and cancerous cells. ${ }^{44}$ In addition, coupled with PIDD, FAS could strengthen BID expression to upregulate cytochrome $c$ through the mitochondrial pathway to trigger the classic apoptosis cascade. ${ }^{44}$ However, the levels of apoptosis were approximately $7 \%$ higher in the PTEN wild-type LN229 cells than in U251 cells, suggesting that the apoptotic response remains partially dependent on PTEN. Aberrant expressed miR-21 in U251 and U87 GBM cell were shown to regulate $\mathrm{p} 53$, TGF- $\beta$, and apoptosis pathway. ${ }^{30}$ These data were consistent with cellular mechanisms that control cellcycle arrest and cancer cell apoptosis, and indicated that miR- 

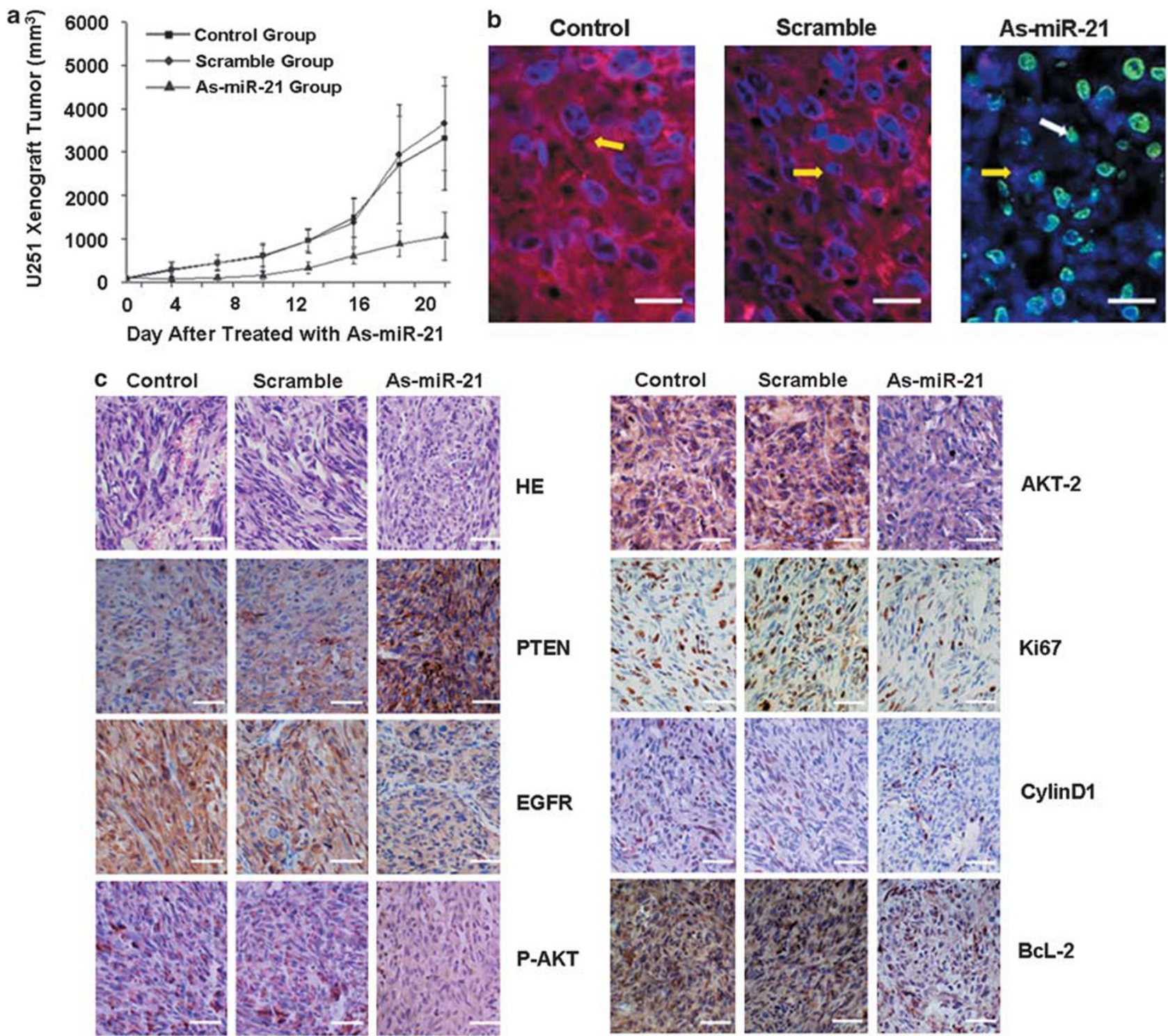

EGFR
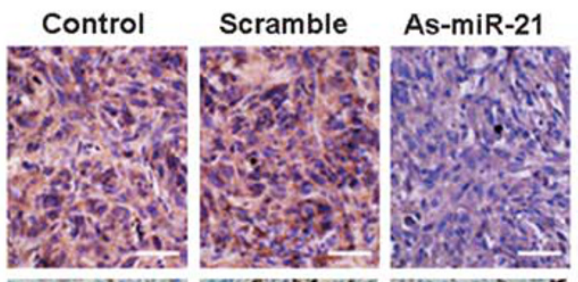

AKT-2
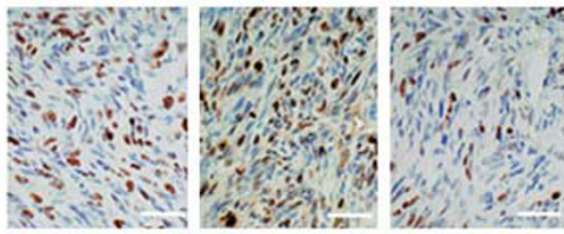

Ki67
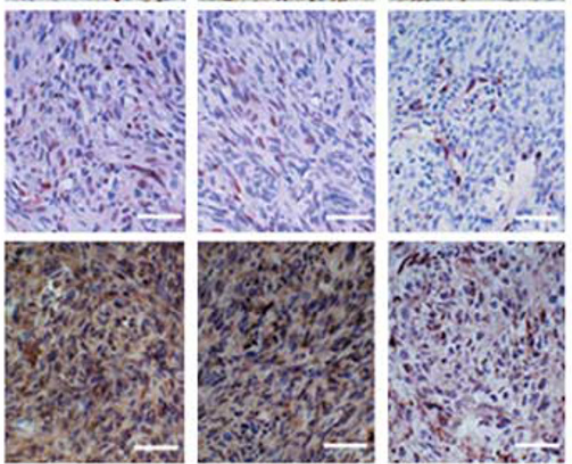

\section{CylinD1}

P-AKT

Figure 5 U251 GBM cell xenograft tumor experiment. (a) Growth of U251 GBM cell xenografts. Inhibition of miR-21 by lipofectamine-mediated As-miR-21 delivery inhibited tumor growth. (b) Apoptosis in xenograft tumors after As-miR-21 treatment. Inhibition of miR-21, as shown by in situ hybridization (red staining), increased apoptosis as determined by TUNEL-positive cells (green staining). Yellow arrow indicates miR-21 in situ expression in U251 xenograft tumor cells. White arrow indicates apoptotic U251 GBM xenograft tumor cells. (c) Immunohistochemistry analysis of U251 xenograft tumors. PTEN expression was increased in U251 xenograft tumors. pAkt, AKT-2, EGFR, cyclin D1, and Bcl-2 expression were suppressed simultaneously. Bar $=100 \mu$ m.

21 could modulate the gene network that associated with the observed phenotype changes.

Further examination of the downstream pathways revealed another interesting observation. Despite non-functional PTEN in U251 cells, the levels of EGFR and activated Akt were inhibited by As-miR-21 treatment, similar to the effects observed in LN229 cells containing wild-type PTEN. EGFR has been reported to be regulated by Stat 3 in a Stat $3 \mathrm{Bcl}-$ 3EGFRStat3 positive signaling loop in EB virus-meditated human cervical carcinoma transformation. ${ }^{45}$ The transcription factor Stat3 was suppressed in this study, consistent with it being predicted to be miR-21 target by mathematic algorithm. ${ }^{46}$ The human miR-21 promoter retains Stat3 binding sites, and its high conservation among vertebrates suggests that highly conserved transcriptional regulatory mechanisms operate on the promoter. Stat3-dependent miR-21 transcription was shown in IL-6-stimulated XG-1 and INA-6 myeloma and HepG2 hepatocellular carcinoma cells. ${ }^{47}$ Further analysis is required to determine whether a similar relationship exists in gliomas.

In summary, we validated that downregulation of miR-21 inhibited the growth of GBM cell lines and induced apoptosis. These effects were only partially dependent on PTEN, highlighting the existence of multiple, and possibly yet unknown, targets of miR-21. Inhibition of miR-21 also suppressed EGFR and Akt activity. These observations were 
confirmed in in vivo xenograft experiments that showed the potential clinical relevance of miR-21-targeting agents. A recent report presented some small-molecule compounds that could suppress miR-21 expression in cancer cells. ${ }^{48}$ Targeting miR-2 1 by antisense or small-molecule compounds may represent new targeted therapeutic strategies for human cancers, including gliomas.

\section{ACKNOWLEDGEMENTS}

This work was supported by the China National Natural Scientific Fund (30971136 and 30872985), the Tianjin Science and Technology Committee (09JCZDJC17600), a Program for New Century Excellent Talents in University (NCET-07-0615), the Oreffice Foundation, and Anthony Bullock Brain Tumor Research Fund (WZ).

\section{DISCLOSURE/CONFLICT OF INTEREST}

The authors declare no conflict of interest.

1. Stewart LA. Chemotherapy in adult high-grade glioma: a systematic review and meta-analysis of individual patient data from 12 randomised trials. Lancet 2002;359:1011-1018.

2. Baulcombe D. DNA events: an RNA microcosm. Science 2002;297: 2002-2003.

3. He L, Hannon GJ. MicroRNAs: small RNAs with a big roll in gene regulation. Nature Gen 2004;5:522-531.

4. Lee RC, Ambros V. An extensive class of small RNAs in Caenorhabditis elegans. Science 2001;294:862-864.

5. Akbergenov R, Si-Ammour A, Blevins $T$, et al. Molecular characterization of geminivirus-derived small RNAs in different plant species. Nucleic Acids Res 2006;34:462-471.

6. Bazzoni F, Rossato M, Fabbri M, et al. Induction and regulatory function of miR-9 in human monocytes and neutrophils exposed to proinflammatory signals. Proc Natl Acad Sci USA 2009;106:5282-5287.

7. Webster RJ, Giles KM, Price KJ, et al. Regulation of epidermal growth factor receptor signaling in human cancer cells by microRNA-7. J Biol Chem 2009;284:5731-5741.

8. Sun $\mathrm{Y}, \mathrm{Wu} \mathrm{J}, \mathrm{Wu} \mathrm{SH}$, et al. Expression profile of microRNAs in c-Myc induced mouse mammary tumors. Breast Cancer Res Treat; 118:185-196. doi: 10.1007/s10549-008-0171-6.

9. Cummins JM, He Y, Leary RJ, et al. The colorectal microRNAome. Proc Natl Acad Sci USA 2006;103:3687-3692.

10. Calin GA, Sevignani C, Dumitru CD, et al. Human microRNA genes are frequently located at fragile sites and genomic regions involved in cancers. Proc Natl Acad Sci USA 2004;101:2999-3004.

11. Calin GA, Dumitru CD, Shimizu M, et al. Frequent deletions and down-regulation of micro-RNA genes miR15 and miR16 at 13q14 in chronic lymphocytic leukemia. Proc Natl Acad Sci USA 2002;99: 15524-15529.

12. Cimmino A, Calin GA, Fabbri $M$, et al. miR-15 and miR-16 induce apoptosis by targeting BCL2. Proc Natl Acad Sci USA 2005;102: 13944-13949.

13. He L, Thomson JM, Hemann MT, et al. A microRNA polycistron as a potential human oncogene. Nature 2005;435:828-833.

14. Mott JL, Kobayashi S, Bronk SF, et al. Mir-29 regulates Mcl-1 protein expression and apoptosis. Oncogene 2007;26:6133-6140.

15. Akao $Y$, Nakagawa $Y$, Naoe T. let-7 microRNA functions as a potential growth suppressor in human colon cancer cells. Biol Pharm Bull 2006;29:903-906.

16. Welch C, Chen Y, Stallings RL. MicroRNA-34a functions as a potential tumor suppressor by inducing apoptosis in neuroblastoma cells. Oncogene 2007;26:5017-5022.

17. Dutta KK, Zhong Y, Liu YT, et al. Association of microRNA-34a overexpression with proliferation is cell type-dependent. Cancer Sci 2007;98:1845-1852.

18. Ciafrè $S A$, Galardi $S$, Mangiola $A$, et al. Extensive modulation of a set of microRNAs in primary glioblastoma. Biochem Biophys Res Commun 2005;334:1351-1358.

19. Chan JA, Krichevsky AM, Kosik KS. Microrna-21 is an antiapoptotic factor in human glioblastoma cells. Cancer Res 2005;65:6029-6033.
20. Zhu S, Si ML, Wu H, et al. MicroRNA-21 targets the tumor suppressor gene tropomyosin 1 (TPM1). J Biol Chem 2007;282:14328-14336.

21. Asangani IA, Rasheed SA, Nikolova DA, et al. MicroRNA-21 (miR-21) post-transcriptionally downregulates tumor suppressor Pdcd4 and stimulates invasion, intravasation and metastasis in colorectal cancer. Oncogene 2008;27:2128-2136.

22. Lu Z, Liu M, Stribinskis V, et al. MicroRNA-21 promotes cell transformation by targeting the programmed cell death 4 gene. Oncogene 2008;27:4373-4379.

23. Frankel LB, Christoffersen NR, Jacobsen A, et al. Programmed cell death 4 (PDCD4) is an important functional target of the microRNA miR-21 in breast cancer cells. J Biol Chem 2008;283:1026-1033.

24. Meng $F$, Henson $R$, Wehbe-Janek $H$, et al. MicroRNA-21 regulates expression of the PTEN tumor suppressor gene in human hepatocellular cancer. Gastroenterology 2007;133:647-658.

25. Kato $\mathrm{H}$, Kato $\mathrm{S}$, Kumabe $\mathrm{T}$, et al. Functional evaluation of p53 and PTEN gene mutations in gliomas. Clin Cancer Res 2000;6:3937-3943.

26. Park CM, Park MJ, Kwak $\mathrm{HJ}$, et al. lonizing radiation enhances matrix metalloproteinase-2 secretion and invasion of glioma cells through Src/epidermal growth factor receptor-mediated p38/Akt and phosphatidylinositol 3-kinase/Akt signaling pathways. Cancer Res 2006;66:8511-8519.

27. You $Y$, Pu P, Huang $Q$, et al. Antisense telomerase RNA inhibits the growth of human glioma cells in vitro and in vivo. Int J Oncol 2006;28:1225-1232.

28. Hosack DA, Dennis Jr G, Sherman BT, et al. Identifying biological themes within lists of genes with EASE. Genome Biol 2003;4:R70.

29. Pu P, Kang C, Li J, et al. The effects of antisense AKT2 RNA on the inhibition of malignant glioma cell growth in vitro and in vivo. J Neurooncol 2006;76:1-11.

30. Papagiannakopoulos T, Shapiro A, Kosik KS. MicroRNA-21 targets a network of key tumor-suppressive pathways in glioblastoma cells. Cancer Res 2008;68:8164-8172.

31. Ruan J, Chen H, Kurgan L, et al. HuMiTar: a sequence-based method for prediction of human microRNA targets. Algorithms Mol Biol 2008;3:16.

32. Guillamo JS, de Boüard $S$, Valable $S$, et al. Molecular mechanisms underlying effects of epidermal growth factor receptor inhibition on invasion, proliferation, and angiogenesis in experimental glioma. Clin Cancer Res 2009;15:3697-3704.

33. Meng $F$, Henson $R$, Lang $M$, et al. Involvement of human micro-RNA in growth and response to chemotherapy in human cholangiocarcinoma cell lines. Gastroenterology 2006;7:2113-2129.

34. Conti A, Aguennouz M, La Torre D, et al. MiR-21 and 221 upregulation and miR-181b downregulation in human grade II-IV astrocytic tumors. J Neurooncol 2009;3:325-332.

35. Krek A, Grün D, Poy MN, et al. Combinatorial microRNA target predictions. Nat Genet 2005;5:495-500.

36. Chen Y, Liu W, Chao T, et al. MicroRNA-21 down-regulates the expression of tumor suppressor PDCD4 in human glioblastoma cell T98G. Cancer Lett 2008;2:197-205.

37. Gabriely G, Wurdinger T, Kesari S, et al. MicroRNA 21 promotes glioma invasion by targeting matrix metalloproteinase regulators. Mol Cell Biol 2008;17:5369-5380.

38. Li Y, Li W, Yang Y, et al. MicroRNA-21 targets LRRFIP1 and contributes to VM-26 resistance in glioblastoma multiforme. Brain Res 2009;1286:13-18.

39. Endersby R, Baker SJ. PTEN signaling in brain: neuropathology and tumorigenesis. Oncogene 2008;27:5416-5430.

40. Leslie NR, Downes CP. PTEN: the down side of PI3-kinase signalling Cell Signal 2002;14:285-295.

41. Kaper F, Dornhoefer N, Giaccia AJ. Mutations in the PI3K/PTEN/TSC2 pathway contribute to mammalian target of rapamycin activity and increased translation under hypoxic conditions. Cancer Res 2006;66:1561-1569.

42. Corsten MF, Miranda R, Kasmieh R, et al. MicroRNA-21 knockdown disrupts glioma growth in vivo and displays synergistic cytotoxicity with neural precursor cell delivered S-TRAIL in human gliomas. Cancer Res 2007;19:8994-9000.

43. Lei K, Davis RJ. JNK phosphorylation of Bim-related members of the Bcl2 family induces Bax-dependent apoptosis. Proc Natl Acad Sci USA 2003;5:2432-2437.

44. Kroemer G, Martin SJ. Caspase-independent cell death. Nat Med 2005; 11:725-730. 
45. Kung $C P$, Raab-Traub N. Epstein-Barr virus latent membrane protein 1 induces expression of the epidermal growth factor receptor through effects on Bcl-3 and STAT3. J Virol 2008;11: 5486-5493.

46. Rehmsmeier $M$, Steffen $P$, Hochsmann $M$, et al. Fast and effective prediction of microRNA/target duplexes. RNA 2004;10: 1507-1517.
47. Löffler D, Brocke-Heidrich K, Pfeifer G, et al. Interleukin-6 dependent survival of multiple myeloma cells involves the Stat3-mediated induction of microRNA-21 through a highly conserved enhancer. Blood 2007;110:1330-1333.

48. Gumireddy $K$, Young DD, Xiong $X$, et al. Small-molecule inhibitors of microRNA miR-21 function. Angew Chem Int Ed Engl 2008;47: 7482-7484. 